\title{
Effect of carboxymethylated cellulose nanofibril concentration regime upon material forming on mechanical properties in films and filaments
}

\author{
Karl M. O. Håkansson $(\mathbb{D}$
}

Received: 18 August 2020/Accepted: 29 October 2020/Published online: 16 November 2020

(C) The Author(s) 2020

\begin{abstract}
It is predicted that the forest and materials from the forest will play an important role to enable the transformation from our linear present to a circular and sustainable future. Therefore, there is a need to understand the materials that can be extracted from the forest, and how to use them in an efficient manner. Here, carboxymethylated cellulose nanofibrils (CNF) from the forest are used to produce films and filaments with the aim to preserve the impressive mechanical properties of a single CNF in a macro-scale material. The mechanical properties of both the films (tensile strength of $231 \mathrm{MPa}$ ) and filaments (tensile strength of $645 \mathrm{MPa}$ ) are demonstrated to be maximized when the
\end{abstract}

starting suspension is in a flowing state. This is a new insight with regards to filament spinning of CNF, and it is here argued that the three main factors contributing to the mechanical properties of the filaments are (1) the possibility to produce a self-supporting filament from a suspension, (2) the CNF alignment inside the filament and (3) the spatial homogeneity of the starting suspension. The results in this study could possibly also apply to other nanomaterials such as carbon nanotubes and silk protein fibrils, which are predicted to play a large part in future high performing applications.
Electronic supplementary material The online version contains supplementary material available at https://doi.org/10. 1007/s 10570-020-03566-z.

K. M. O. Håkansson ( $₫)$

RISE Bioeconomy \& Health, Drottning Kristinas väg 61

B, 11428 Stockholm, Sweden

e-mail: karl.hakansson@ri.se 


\section{Graphic abstract}

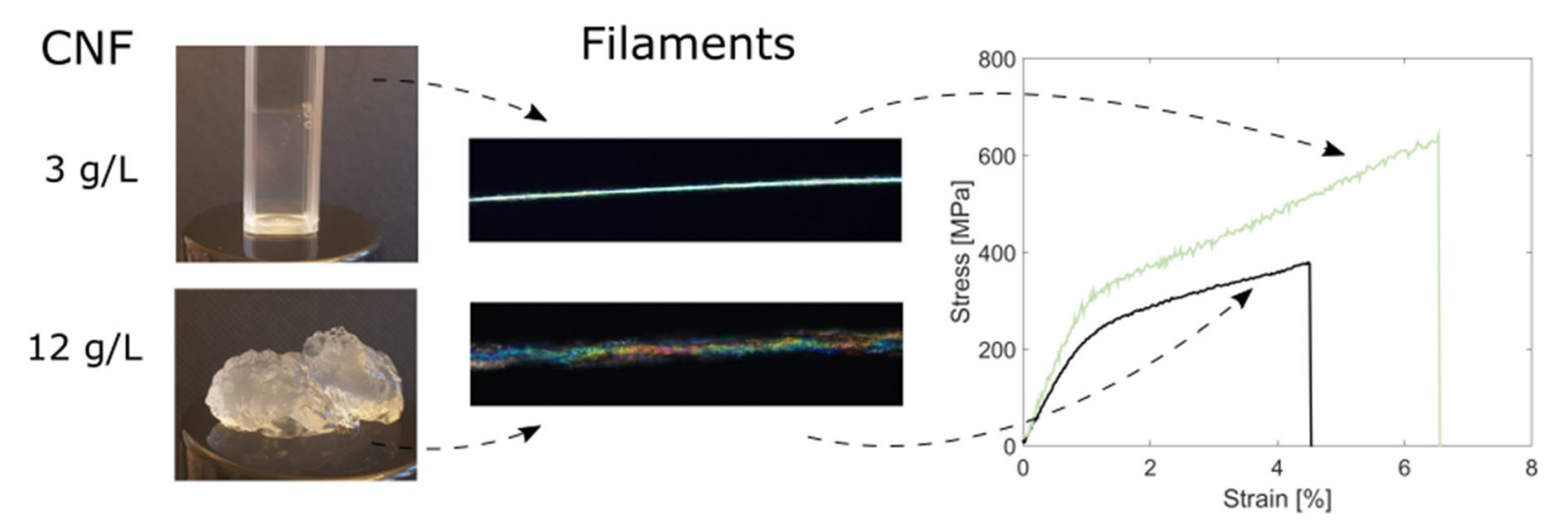

Keywords Cellulose nanofibril · Films - Filament · Spinning $\cdot$ Material manufacturing

\section{Introduction}

As the unique properties of single nanoparticles are increasingly explored, the interest in utilization of the particles and their properties on a macro scale follows. Therefore, the process of assembling the nanoscale building blocks is of importance. Recently, it was proven that the mechanical properties of cellulose nanofibrils (CNF), a nanomaterial with impressive mechanical properties, can be retained in a macroscopic filament by controlling the multiscale assembly of the starting material (Mittal et al. 2018). However, the complete understanding of why this was possible, or maybe why other studies have failed, is still lacking.

CNF is produced by several different organisms on our planet, for example trees, plants and bacteria, see Eichhorn et al. (2009) and Klemm et al. (2011) for an overview. During the synthesis, a large number of glucan chains are produced in parallel by terminal complexes, in vascular plants called rosettes (Saxena and Brown 2005). The glucan chains are thereafter aligned and associated to form the CNFs and crystalize to the cellulose I structure. The thickness of the elementary CNF can vary between organisms from the common 3-4 nm in wood to the more extreme $60 \mathrm{~nm}$ in some algae (Saxena and Brown 2005). Here, the focus is on CNF from wood and, in wood, CNF is the building block responsible for the mechanical properties of the trees, enabling them to grow tall and at the same time withstand high winds (Dinwoodie 2000). In wood, the CNF is integrated with lignin and hemicelluloses, where the lignin acts as the matrix component and hemicellulose is the bonding between the lignin and cellulose (Dinwoodie 2000). As a renewable and abundant material, and due to the inventions of energy efficient liberation processes from wood (Pääkkö et al. 2007; Saito et al. 2006; Wågberg et al. 1987) it is predicted that CNF will be an integral part in the transformation to a circular bioeconomy. The liberated CNFs are either the elementary CNF or aggregates thereof resulting in elongated particles with widths of 4-20 nm and lengths of $0.2-3 \mu \mathrm{m}$, see Nordenström et al. (2017) and Usov et al. (2015). While the elementary CNF in the wood have rather well determined size, it is difficult to liberate all elementary CNFs in a uniform manner and a large distribution of sizes are common in CNF suspensions (Klemm et al. 2018). Moreover, residual fiber fragments are common after the liberation process, resulting in heterogeneous suspensions with particles sizes ranging from $\mathrm{nm}$ to $\mathrm{mm}$ (Foster et al. 2018). Furthermore, the variety of different pre-treatments and mechanical delamination processes results in a plethora of different CNF qualities, varying in both chemical and mechanical properties (Klemm et al. 2011). Elucidating how the CNF properties, such as size, stiffness, residual fiber fragment proportion and chemical composition influence the static and dynamic bulk properties of the corresponding suspensions, and the time dependency thereof, is an active research field with many unknowns (Brouzet et al. 2018, 2019; Rosen et al. 2020). Alongside the studies of connecting the CNF properties with the suspension properties, 
the suspensions are used and formed into 1D filaments, 2D films and 3D objects. Making the connection from CNF extraction method, through CNF properties and suspension properties, via forming process to the properties of the final material is of course the final goal, however, many small steps must be taken to get there. Here, the focus will be on connecting suspension properties to filament and film properties of carboxymethylated CNF.

The idea with filament spinning is to create 1D filaments by forcing a flowing material (usually polymer or particle in a melt or solution) through a spinneret (nozzle with many holes) (Denn 1983). The spinneret is designed to produce the wanted size and shape of the filament, and when the flowing material exits the spinneret the material must solidify (stop flowing) to retain the filament shape state. This solidification can be achieved with a change from liquid to solid by a temperature decrease as in melt spinning or coagulation by changing the chemical environment as in wet spinning. In order to achieve optimized properties of the formed filament, the structure and homogeneity inside the filament is crucial (Denn 1983). The starting material is usually anisotropic, such as a long polymer, and therefore the alignment of the polymer inside the filament determines the properties of the filament itself. Furthermore, the alignment can be optimized by stretching (drawing) of the filament at different stages of the process (Denn 1983). Spinning more exotic materials may require more exotic spinning techniques, for example liquid crystal spinning (Behabtu et al. 2013) or gel spinning and recently a lot of work has been going into mimicking the spider silk spinning (Kinahan et al. 2011).

Several studies have focused on filament spinning of CNF, varying concentration, starting material, nozzles and have relied on different effects for the solidification such as the shear thinning effect of viscoelastic solids (network rupture and reformation), screening of the surface charges and interfacial polyelectrolyte complexation (Geng et al. 2017; Ghasemi et al. 2017; Hooshmand et al. 2015, 2017; Håkansson et al. 2014; Iwamoto et al. 2011; Kafy et al. 2017; Kim et al. 2019; Lundahl et al. 2016; Mertaniemi et al. 2016; Mittal et al. 2017, 2018, 2019; Mohammadi et al. 2017; Nechyporchuk et al. 2018; Shen et al. 2016; Toivonen et al. 2017; Torres-Rendon et al. 2014; Walther et al. 2011; Yao et al. 2017). The mechanical properties of the filaments have varied, but the two main conclusions are, firstly, that by increasing the CNF alignment, the ultimate tensile strength and tensile stiffness is also increasing, while the strain at break is decreasing. The second conclusion is that increasing the alignment by drawing after filament formation results in an increased CNF alignment and tensile stiffness, while the strength is maintained and the strain at break is decreased.

Films, on the other hand, are often produced and used as a characterization method of the $\mathrm{CNF}$, where the mechanical properties of the films are evaluated by tensile testing (Aulin et al. 2010; Fujisawa et al. 2011; Fukuzumi et al. 2009; Henriksson et al. 2008; Syverud and Stenius 2008), providing insights of the starting material. The films are usually produced from a concentration $(<2 \mathrm{~g} / \mathrm{L})$ in water were the particles have high mobility, i.e. not restricted by other particles and well dispersed in space. In this way, films with spatially homogeneous and isotropic structure in 2D of the CNF can be produced and the effect of production process, spatial agglomeration and fibril alignment is canceled (Syverud and Stenius 2008).

In contrast to the CNF lab scale film production, when the aim is to produce 1D CNF filaments, a higher concentration is usually used, since the filament must support itself directly after the filament is formed and the particles must therefore be in contact. But also, when producing films on an industrial continuous scale, an increased concentration is wanted, since any removal of water is time consuming and energy demanding. It is therefore important to understand how the starting concentration of the suspension affects the properties of the final filaments and films.

To understand suspensions of elongated particles, two threshold concentrations are of importance, firstly, the overlap concentration (Geng et al. 2018; Naderi et al. 2014a) and secondly, the concentration where all particles are in continuous contact and a connected network is formed (Naderi et al. 2014a, b). When a network is formed the suspension stops flowing and by the nomenclature adopted from Nordenström et al. (2017), this state is here called Volume Arrested State (VAS), while below this concentration the suspension is called flowing. The overlap concentration, where the CNF starts to interact with neighboring particles is low and could be as low as $0.5-0.6 \mathrm{~g} / \mathrm{L}$ (Geng et al. 2018), due to the large aspect ratio of CNF. The concentration where a VAS is formed is also highly 
affected by the aspect ratio and can be $1.5-7 \mathrm{~g} / \mathrm{L}$ depending on fibril length and charge (Nordenström et al. 2017). The mobility of the CNFs in the suspension can be related to the thresholds. Below the overlap concentration, the CNFs are freely translating and rotating without hindrance, while over the VAS concentration the CNF is locked in a network and are not free to move at all. The concentration regime between the two thresholds is the most complicated, where the particles mobility is reduced but a well dispersed system is still possible to achieve (Nordenström et al. 2017). Note that it may be possible to disrupt the VAS by applying external forcing by fluid flow fields or electric fields and that the disruption could either be global or local. When the VAS is disrupted the suspension can start to flow again. The reformation of the connected VAS after the forcing has been turned off will lead to several networks or one uniform network being formed making the history of the material important. Hence, when processing CNF at a concentration where the particles can interact, extra consideration and characterization must be put into understanding the structure and spatial uniformity. It is in the middle regime that most films are produced, but there is no limitation to using a lower concentration. While filaments must be produced above the overlap concentration and have been produced both in the middle regime and at concentrations where the CNFs are locked in a VAS. How important the starting concentration is when producing filaments and films have not yet been completely understood, and even though concentration variations have been made, they have been focused on either a variation under the VAS transition (Mittal et al. 2019) or a variation over the VAS transition (Lundahl et al. 2016). Therefore, films and filaments will be produced and a comparison with a flowing concentration and a VAS will be presented here.

To summarize, filaments and films from CNF have the potential to be a large part of the transition into a circular bioeconomy necessary for a sustainable resource use. CNF extracted from wood can vary in shape, size, stiffness and surface modification depending on the production process and investigations into how the CNF properties affect the suspensions are ongoing. The concentration has clear effect on the behavior of a CNF suspension due to the elongated shape of the CNF, resulting in different degrees of entanglement at different concentration. However, the full understanding of how the starting concentration is affecting filament and film properties is lacking. This study is therefore aimed at investigating the effects on the mechanical properties of filaments and films at different starting concentration regimes. Two concentrations, in two different concentration regimes, namely in the flowing state and in the VAS are chosen. The suspensions are studied with rheological measurements and polarized optical microscopy (POM) on scales much larger than that of an individual $\mathrm{CNF}$, which in turn are related to the mechanical properties of the films and filaments. This, seemingly simple, parameter variation highlights the need to increase the understanding of concentrated suspensions of nanoparticles in order to optimize the production processes towards performance and economic feasibility.

\section{Results}

The results section is divided into three characterization parts, starting with the three suspensions, followed by the films and finally the filaments.

\section{Suspensions}

A carboxymethylated CNF was used for all trials and to make sure that the same material is used, one batch of carboxymethylated pulp was homogenized at $5 \mathrm{~g} / \mathrm{L}$ and thereafter either concentrated by evaporation or diluted with magnetic stirrer. The goal was to prepare material at two different concentrations, one where the suspension is flowing and the CNFs are mobile and one non-flowing, where the CNFs are locked in a VAS. Three samples were prepared according to Fig. 1a, two flowing samples, 1 and 3 , at $3 \mathrm{~g} / \mathrm{L}$ and one, sample 2 , in a VAS at $12 \mathrm{~g} / \mathrm{L}$. To investigate if the concentration process (evaporation) induces any permanent changes to the material, sample 2 was diluted with a magnetic stirrer resulting in sample 3 , see Fig. 1 .

The rheological properties $G^{\prime}$ and $G^{\prime \prime}$ were determined for the three samples, see Fig. 1b. If $\mathrm{G}^{\prime \prime}$ is much larger than $G^{\prime \prime}$ the fluid is defined as a viscoelastic solid (non-flowing in our case), while if the opposite is true $\left(G^{\prime}\right.$ smaller than $\left.G^{\prime \prime}\right)$ the suspension behaves like a liquid and is flowing (Geng et al. 2018; Naderi et al. 2014a). From the rheological measurements it can be determined that the two samples at $3 \mathrm{~g} / \mathrm{L}$ where $\mathrm{G}^{\prime} \approx$ 


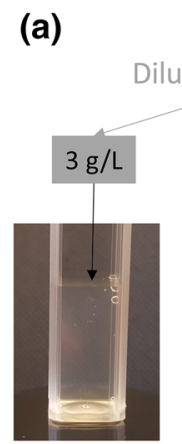

1

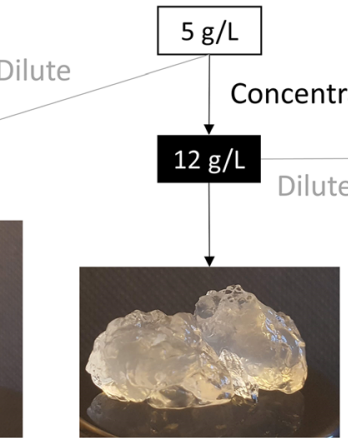

2

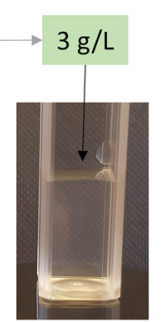

3

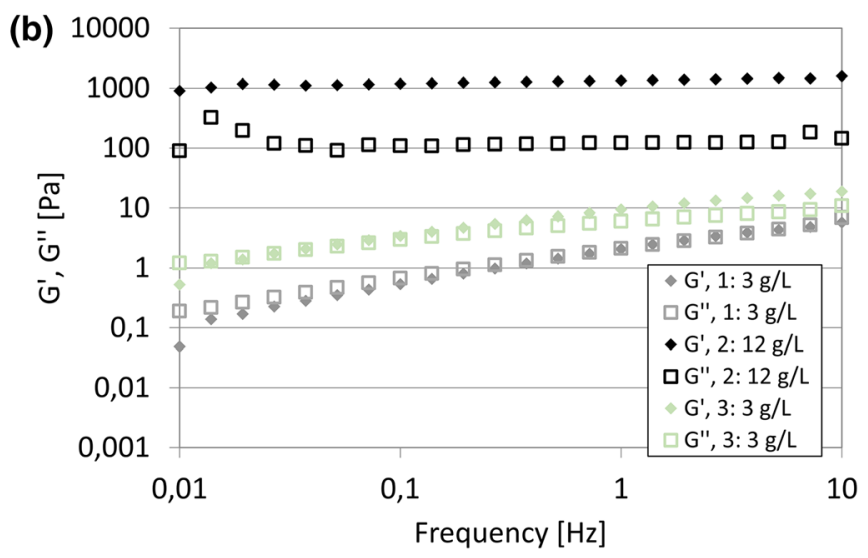

Fig. 1 a Images of the three starting suspensions and a schematic of the dilution and concentration routes. b Rheological measurement, $\mathrm{G}^{\prime}$ and $\mathrm{G}^{\prime \prime}$, of the there samples in $\mathbf{a}$

$\mathrm{G}^{\prime \prime}$ or $\mathrm{G}^{\prime}<\mathrm{G}^{\prime \prime}$ are on the limit to behaving more liquid like, while sample 2 at $12 \mathrm{~g} / \mathrm{L}$ is a viscoelastic solid since $G^{\prime}>G^{\prime \prime}$. With the additional fast test of turning the sample up-side-down, it is concluded that sample 1 and 3 are flowing suspensions, while sample 2 is in a VAS and not flowing. For the full study of the rheological response with varying concentrations of CNF the reader is referred to Naderi et al. (2014a, b). Note that the evaporation followed by a dilution (sample 3) results in an increase in viscosity compared with the original (sample 1), which may be due to an irreversible aggregation of some part of the material or a slight concentration difference, the suspension is nevertheless flowing.

The three different samples were studied with POM and Fig. 2a-c shows one typical image of the each, also found in the supplementary information as larger figures, Figs. S1-S3. At the flowing concentration only a few fiber fragments are visible, while lighter and darker regions are visible for the concentrated sample. The light and dark regions is an indication that the sample is not homogeneous on a scale much larger than the CNF themselves. Finding the exact reason for the light and dark regions in the sample will not be perused here, however, for light to be able to pass through both polarizers the sample must be anisotropic, indicating that the CNF has different preferred alignment in the light and dark regions. Note that the regions are stable in time for sample 2, which, together with the rheological measurement is evidence for the that the CNFs are locked in a VAS due to the high degree entanglement (Geng et al. 2018; Naderi et al. 2014a; Nordenström et al. 2017). For sample 1 and 3, the large fiber fragments are observed to move, further indicating that the system is flowing, and the CNFs are mobile at this concentration.

By image analysis of the POM images the inhomogeneities can be quantified. It is possible to borrow knowledge from the neighboring field of papermaking, where the spatial distribution of fibers in suspensions and dry paper have been studied extensively pioneered by Mason (1950). In papermaking, the spatial distribution of fibers is quantified by the Formation number, lower number means more homogeneous (Johansson and Norman 1996). In this case, the POM formation is determined from the images with the software STFI Mottling Expert. The software performs a Fourier transformation of the images in 2 dimensions and outputs the deviation from the mean for different intervals corresponding to wavelengths in the image (Gonzalez and Woods 1993). In Fig. 2d, the results are displayed, where the dashed lines correspond to the suspensions for the three samples. Sample 1 and 3 are seen to have very low POM formation for all length scales, while sample 2 have increasing variation in the interval from 10 to $150 \mu \mathrm{m}$. The total POM formation number for the samples 1-3 are $3.5 \pm 0.9,31 \pm 4.9$ and $2.5 \pm 0.4$, respectively.

Summarizing, suspension 1 and 3 are flowing and homogeneous while suspension 2 is in a VAS, not flowing and not homogeneous at rest. 
Suspension
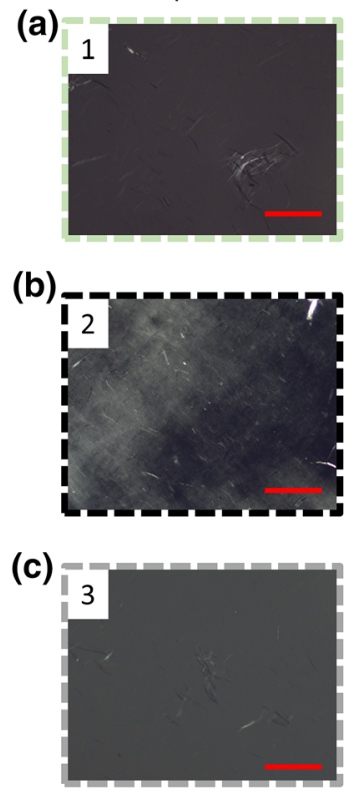

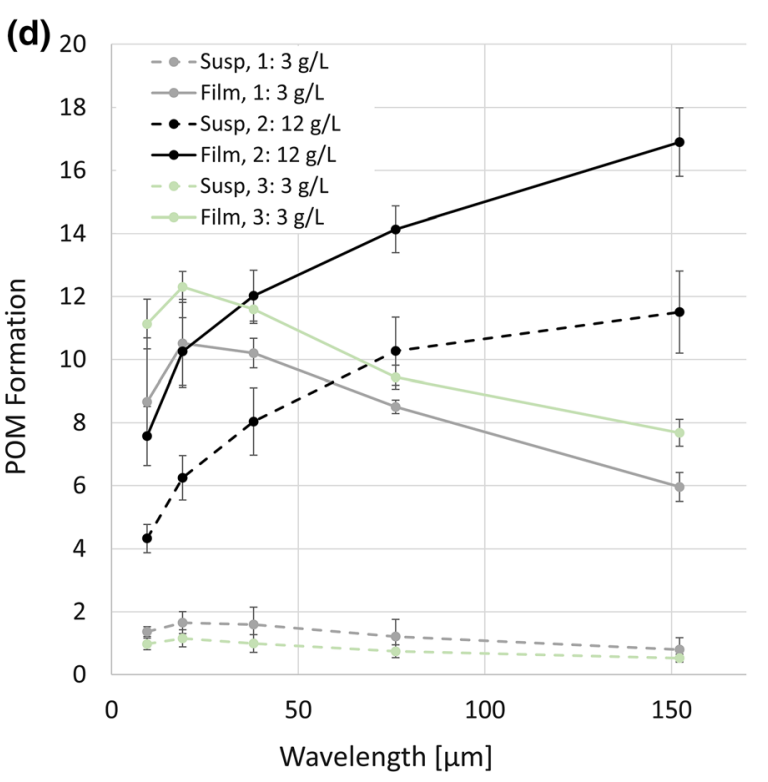

Film

(e)

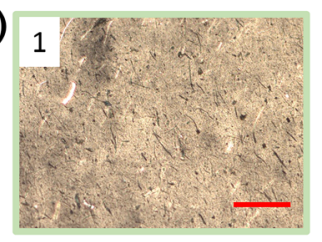

(f)

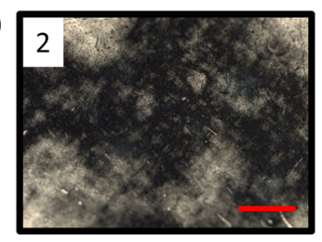

(g)

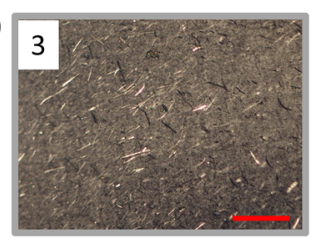

Fig. 2 POM images of the suspensions in a-c, corresponding to sample 1-3, respectively. In d, the POM Formation at different wavelengths (length scales) are presented for both the

Films

The three samples were used to produce films, which were characterized with POM and tensile tests. The films were produced in two slightly different processes. Vacuum filtration was used for the flowing samples while a mild centrifugation step was included to shape the VAS and remove bubbles. Furthermore, a Teflon coated knife was used to cut off the top of the VAS to further shape the VAS to a film, see the experimental section for details. The influence of these extra processing steps will be discussed later. Note that in the literature, when studying the mechanical properties of films, the films are produced from a concentration were the suspension is flowing (Henriksson et al. 2008). The reason for using a low concentration being both that it is easier to form an even film and to avoid spatial variations caused by agglomeration, extensively studied within the paper making field (Kerekes and Schnell 1992; Mason 1950). However, in an industrial film production process, the highest concentration possible without loss of properties is wanted, since removal of water is both time and energy consuming, motivating this study. suspensions (dashed) and the films (solid). In e-g, one representative POM image of the films from sample 1-3 are shown. The scale bar corresponds to $100 \mu \mathrm{m}$ in all images

The films were imaged with POM and representative images are presented in Fig. 2e-g, also these POM images can be found in the supplementary information as larger images, Fig S4-S6. Sample 1 and 3 , show fiber fragments while in sample 2 a similar pattern as its corresponding starting material with light and dark patches are visible. In Fig. 2d, the solid lines correspond to the POM formation of the films. The films from sample 1 and 3 are similar, with a maximum at a wavelength of $10-20 \mu \mathrm{m}$, which corresponds well to the width of the fiber fragments. Interestingly, the film and the suspension of sample 2 are very similar with the difference being a shift to higher values for the film. This is an indication of that the same structures (in the range 10-150 $\mu \mathrm{m}$ ) present in the starting material also exist in the dry film.

Tensile tests were performed with the three different samples and one typical stress-strain curve per sample is displayed in Fig. 3, while Table 1, contains the extracted mean mechanical properties and POM formation. All stress-strain curves are found in the supplementary information, Figs. S7-S9. The films produced from the starting material in the VAS state (sample 2) is seen to have slightly lower stiffness, decreased strength and also larger variation of strength 


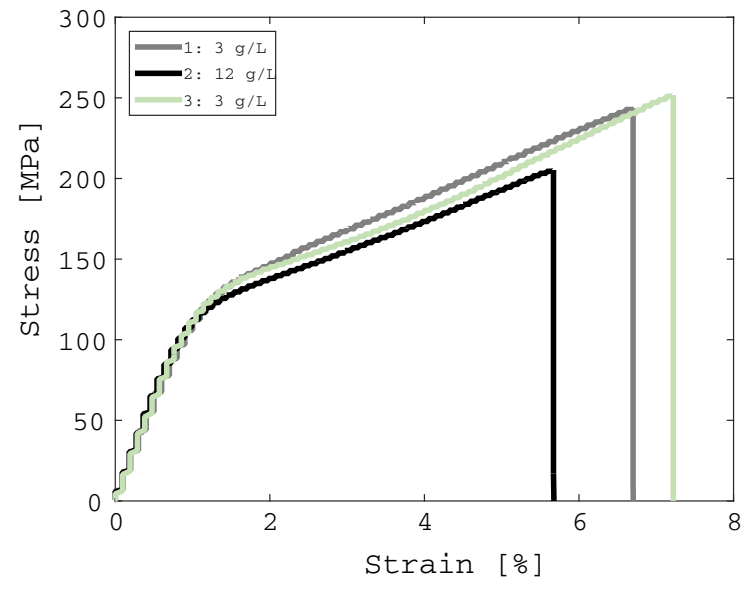

Fig. 3 One representative stress-strain curve each for films produced from the three samples

compared with sample 1 and 3. The total POM formation number presented in Table 1, gives a quantification of the spatial variations in the film and is almost doubled for sample 2 compared to sample 1 and 3 , where a low number correspond to low variation. More investigations regarding the POM formation number must be performed to establish the connection to mechanical properties, but it is here introduced as a potential characterization tool for CNF films. In summary, the films show lower mechanical properties and larger spatial variation for the VAS sample compared to the flowing samples. Where the spatial variations may stem from will be discussed in the discussion section of this manuscript.

Filaments

When the aim is to extract the true potential of a onedimensional nanoparticle into a macroscopic material, aligning and manufacture of a one-dimensional filament is often chosen. Here, filaments are produced by taking advantage of the solidification process of screening the surface charges of the CNF (Fall et al. 2011) in a similar manner as has previously been used to spin filaments with the flow focusing nozzle (Håkansson et al. 2014; Mittal et al. 2017, 2018; Nechyporchuk et al. 2018). By ejecting the starting material from a nozzle into a bath with low $\mathrm{pH}$ a strong enough gel filament is produced to enable further processing, in this case drying. Using a syringe nozzle instead of the flow focusing nozzle makes it possible to use not only the flowing suspension, but also the starting material in a VAS state.

Optical microscopy, POM and scanning electron images are shown in Fig. 4. The filaments produced from sample 1 and 3 are seen to be straight and homogeneous, while the filaments from sample 2 are not.

A typical stress-strain curve is displayed for each case in Fig. 5a, all results are compiled in Table 2 and all curves can be seen in the supplementary information, Figs. S10-S12. The tensile results are similar to the film case, the material in the VAS state results in less stiff and strong filaments. However, compared with the films, both the stiffness and strength are higher for the filaments. As stated in the introduction, the stiffness and strength are highly correlated to the alignment. Therefore, the fibril alignment was measured with Wide Angle X-ray Scattering (WAXS) and the results shown in Fig. 5b; Table 2. With all spinning conditions constant, the non-flowing suspension resulted in less aligned CNF. The alignment is related to how easy it is for the CNFs to be oriented by a fluid flow, which is directly related to the concentration. This may be proposed to be the reason for the discrepancy in mechanical data, but in the next section a case will be made for that the alignment is not the sole reason.

Table 1 Mechanical properties and POM Formation of the films. At least six films for each sample were measured

\begin{tabular}{llllll}
\hline Film sample $(\mathrm{g} / \mathrm{L})$ & Grammage $(\mathrm{g} / \mathrm{m})$ & Tensile strength $(\mathrm{MPa})$ & Tensile modulus $(\mathrm{GPa})$ & Strain at break $(\%)$ & POM formation \\
\hline $1: 3$ & 28 & $225 \pm 23$ & $12.7 \pm 0.5$ & $5.5 \pm 1.1$ & $22 \pm 2.4$ \\
$2: 12$ & 68 & $173 \pm 42$ & $12.0 \pm 0.4$ & $4.6 \pm 2.2$ & $57 \pm 6.2$ \\
$3: 3$ & 42 & $231 \pm 28$ & $12.6 \pm 0.5$ & $6.0 \pm 1.3$ & $30 \pm 1.9$ \\
\hline
\end{tabular}


1
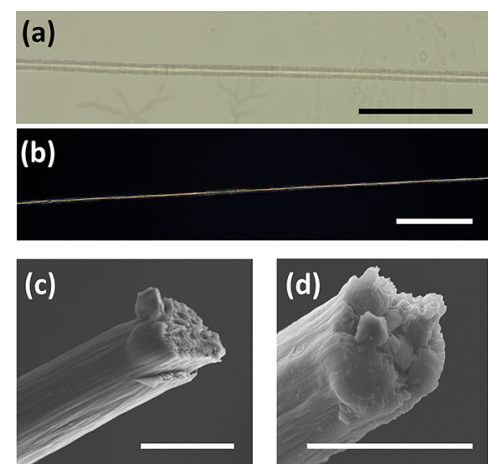

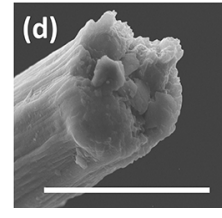

2
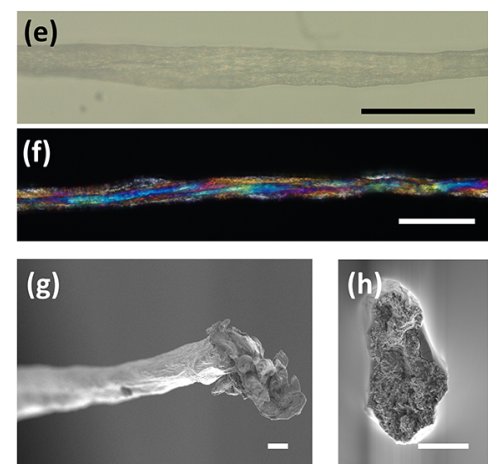

3

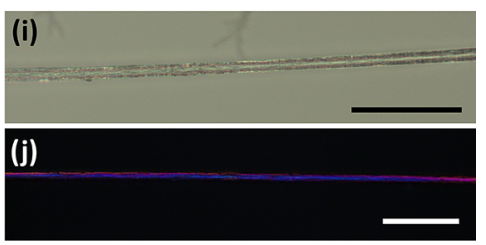

(I)

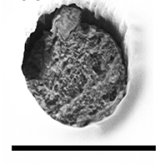

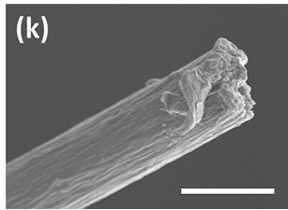

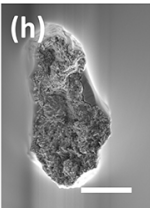

Fig. 4 Filaments made from sample 1 is seen in a-d, sample 2 in $\mathbf{e}-\mathbf{h}$ and sample 3 in $\mathbf{i}-\mathbf{l}$. One optical microscopy image (a, e, $\mathbf{i}$ ), one POM image b, f, j and two SEM images c, d, g, h, k, l of

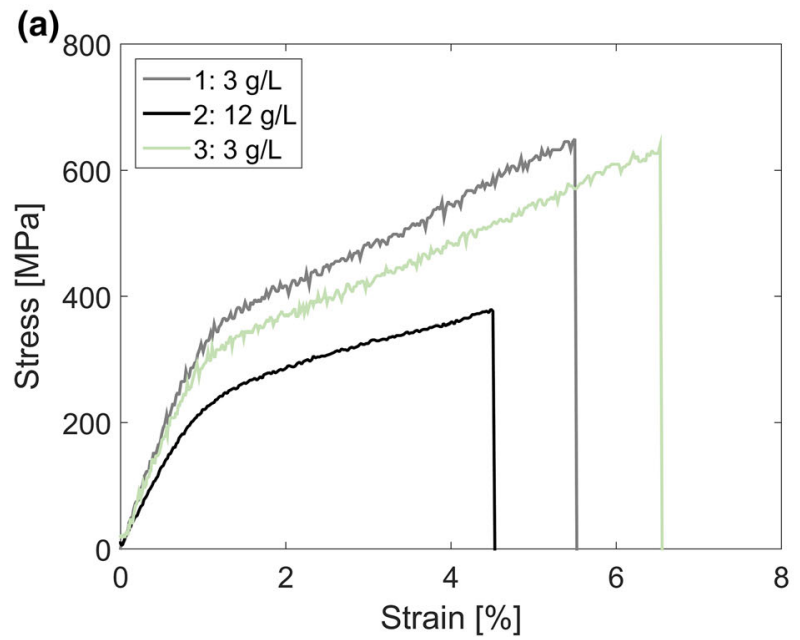

Fig. 5 a Representative stress-strain curves for filaments produced from the three samples are shown. $\mathbf{b}$ the insert is a WAXS diffractogram and the azimuthal integration of the filaments are presented. The scale bar in $\mathbf{a}, \mathbf{b}, \mathbf{e}, \mathbf{f}, \mathbf{i}$ and j represent $100 \mu \mathrm{m}$, while the scale bar in $\mathbf{c}, \mathbf{d}, \mathbf{g}, \mathbf{h}, \mathbf{k}$ and l represent $10 \mu \mathrm{m}$

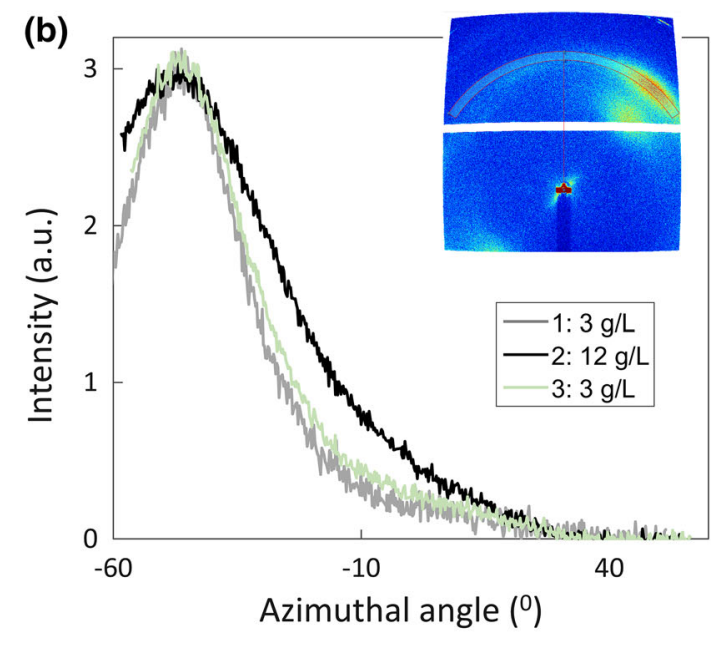

(200)-peak for the three samples are presented in the graph, showing the alignment of the cellulose chains

Table 2 Mechanical properties and alignment of the filaments. At least ten filaments for each sample were measured

\begin{tabular}{lllllll}
\hline $\begin{array}{l}\text { Filament sample } \\
(\mathrm{g} / \mathrm{L})\end{array}$ & $\begin{array}{l}\text { Diameter } \\
(\mu \mathrm{m})\end{array}$ & $\begin{array}{l}\text { Tensile strength } \\
(\mathrm{MPa})\end{array}$ & $\begin{array}{l}\text { Tensile modulus } \\
(\mathrm{GPa})\end{array}$ & $\begin{array}{l}\text { Strain at break } \\
(\%)\end{array}$ & $\begin{array}{l}\text { Orientation index } \\
(\mathrm{fc})\end{array}$ & $\begin{array}{l}\text { Order parameter } \\
(\mathrm{S})\end{array}$ \\
\hline $1: 3$ & 8.5 & $645 \pm 73$ & $34 \pm 1.3$ & $5.2 \pm 0.9$ & 0.78 & 0.56 \\
$2: 12$ & 21 & $381 \pm 77$ & $23 \pm 2.6$ & $4.8 \pm 1.8$ & 0.65 & 0.52 \\
$3: 3$ & 8.5 & $631 \pm 56$ & $31 \pm 1.9$ & $6.3 \pm 0.6$ & 0.76 & 0.56 \\
\hline
\end{tabular}




\section{Discussion}

Remember that the films produced from the flowing state resulted in higher mechanical properties and more homogeneous films than the films produced by the VAS state. Since the material is the same in both cases while the film properties are different it is concluded that the structuring of the CNF is the difference between the film samples, which was also detectable in the POM images. The question is then raised why the structure is different and in which process/processes this structure was set. For the VAS case, three extra processing steps have been added compared with the flowing case. The added processing steps are; (1) water was evaporated during magnetic stirring, (2) the VAS was centrifuged to shape the VAS and remove bubbles and lastly (3) the top of the VAS was removed with a Teflon coated knife. The third process of removing additional material is not likely to affect the interior network structure since the VAS is known to be easily disrupted (Torres-Rendon et al. 2014) and the knife is only affecting the top surface. The second, centrifugation step, has been studied in order to remove larger particles (Naderi et al. 2014a) from CNF suspensions. It was concluded that at concentrations as low as $1.8 \mathrm{~g} / \mathrm{L}$, no pellet is formed, and the concentration of the supernatant was only a few percent higher the concentration before the centrifugation. This is an indication that there are little effects on the CNF network from the centrifugation at the high concentration of $12 \mathrm{~g} / \mathrm{L}$. However, the experiments presented here show that structural inhomogeneities are introduced during first process; the evaporation and magnetic stirring. These structures are also visible in the film and it is therefore hypothesized that the main structural changes are introduced during the evaporation process. In other words, CNF networks are formed during the evaporation from $5 \mathrm{~g} / \mathrm{L}$ to $12 \mathrm{~g} / \mathrm{L}$ that are present also in the final films since they do cannot self-diffuse into one homogeneous network.

At a first glance at the filament results, it is tempting to attribute the decreased mechanical properties of the filaments, produced from the higher concentration, to the fact that the CNFs are less aligned. Further, that the CNFs are less aligned due to the connected network formed at an increased concentration, which would lead to that the fluid flow is not able to align the particles to the same extent as for the low concentration cases. The argument for the cause of lower alignment is valid. However, the first clue to why the alignment alone does not explain the reduced mechanical properties is the strain at break (see Table 2) which at a lower alignment should be higher, rather than lower as in this case. The second clue comes from the comparison with published data of filaments produced from CNF. Most studies where filaments from $\mathrm{CNF}$ are produced have presented tensile data in the form of tensile stiffness and ultimate tensile strength and that will therefore be used for comparison, see Fig. 6. Different starting materials were used, such as non-pretreated, TEMPO, carboxymethylated, enzymatic, and the concentrations ranged from 3 to $70 \mathrm{~g} / \mathrm{L}$, (Geng et al. 2017; Ghasemi et al. 2017; Hooshmand et al. 2015, 2017; Håkansson et al. 2014; Iwamoto et al. 2011; Kafy et al. 2017; Kim et al. 2019; Lundahl et al. 2016; Mertaniemi et al. 2016; Mittal et al. 2017, 2018; Mohammadi et al. 2017; Nechyporchuk et al. 2018; Shen et al. 2016; Toivonen et al. 2017; Torres-Rendon et al. 2014; Walther et al. 2011; Yao et al. 2017). Furthermore, the solidification mechanism varies from blocking the electrostatic repulsion, interfacial polyelectrolyte complexation to a concentration increase. All the differences in the preciously performed studies make it difficult to compare the studies in a structured way, however, by highlighting the studies which explicitly stated that the starting material was at a concentration were the suspension was flowing and below the VAS concentration some insights can be gained. Most studies do not present the rheological response or the homogeneity of their starting material, nor do they discuss the homogeneity of the final filament. Therefore, the studies that positively state that the CNF is in the dispersion state are indicated with green squares ( $\sim 3 \mathrm{~g} / \mathrm{L}$ ), and all others, with concentrations from 8 to $70 \mathrm{~g} / \mathrm{L}$ are blue circles.

In Fig. 6, a dashed line is drawn connecting the strongest filaments at the highest and lowest stiffness, representing the strength possible to achieve at a given stiffness. Both the CNF alignment and humidity are known reasons for the combined increase in both stiffness and strength (Mittal et al. 2018). The green markers end up close to the dashed line, while the blue markers are scattered below the line, with one exception (Kim et al. 2019). The experiments presented in this study are indicated by diamonds, numbers 1-3 and are also color coded in the same 


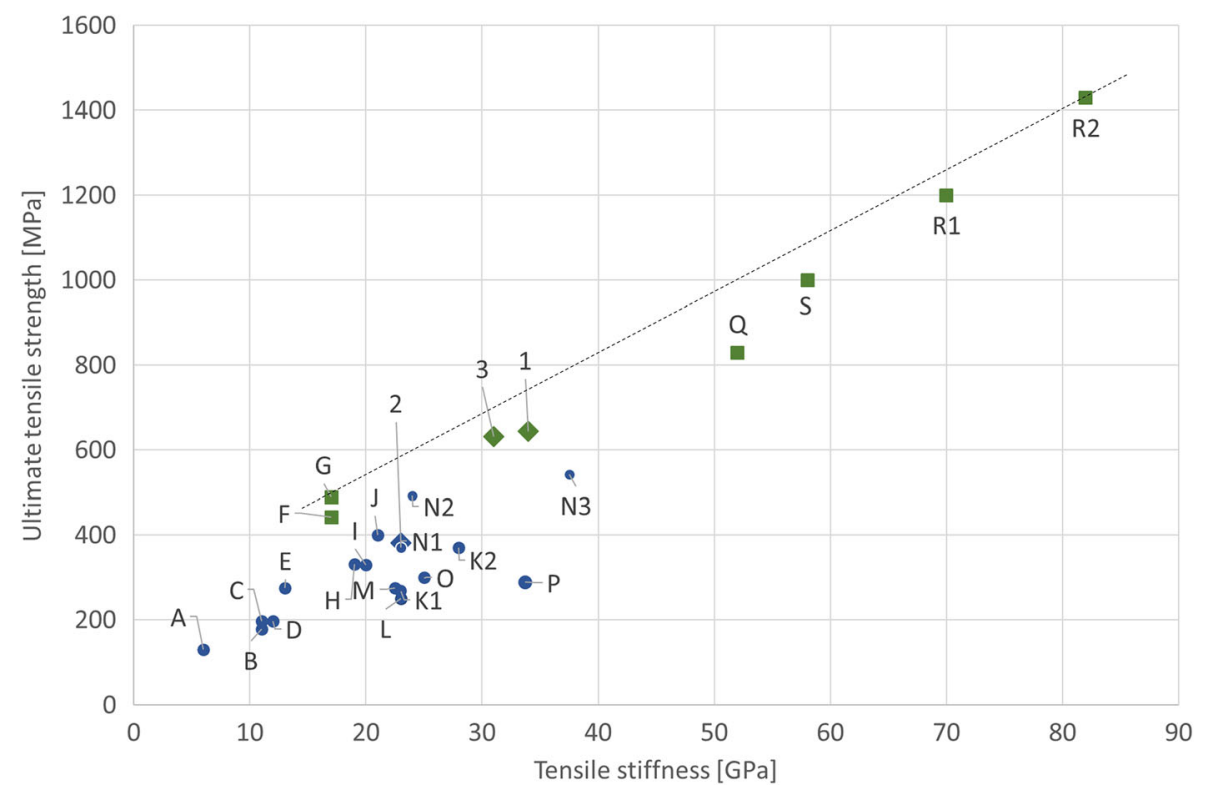

A Ghasemi et al. 2017

B Hooshmand et al. 2017

C Hooshmand et al. 2015

D Yao et al. 2017

E Mertaniemi et al. 2016

F Nechyporchuk et al. 2018

G Håkansson et al. 2014

H Iwamoto et al. 2011

I Mohammadi et al. 2017

J Kafy et al. 2017

K Geng et al. 2017

L Toivonen et al. 2017

M Walther et al. 2011

N Kim et al. 2019

O Lundahl et al. 2016

P Torres-Rendon et al. 2014

Q Mittal et al. 2017

R Mittal et al. 2018

S Mittal et al. 2019

Fig. 6 Ultimate tensile strength versus tensile stiffness for filaments spun from CNF

manner as the values found in the literature. The samples produced from the flowing suspension end up close to the dashed line, while the suspension in the VAS state end up just below $400 \mathrm{MPa}$. This is the clue that tells us that the alignment is not the complete explanation, since at the stiffness of $23 \mathrm{GPa}$, the straight line predicts it to be possible to produce filaments with tensile strengths above $500 \mathrm{MPa}$. Therefore, the in-homogeneity detected in the starting material and in the final filament is concluded to be the reason for the filaments not achieving their full potential, in the same way as for the film case. This leads to the question; have all studies in blue not reached their full potential simply due to a too high concentration has been used, where inhomogeneities already are present in the starting suspension?

Two studies with concentration variations are worth mentioning, firstly, Lundahl et al. (2016) showed how the mechanical properties decreased with $50-70 \%$ as the concentration of the spinning suspension was increased from 20 to $70 \mathrm{~g} / \mathrm{L}$. This decrease was not correlated to alignment and a complete explanation was not found. Secondly, and with opposite results, Mittal et al. (2019) observed a 30\% increased stiffness and strength with increasing concentration from 2 to $3 \mathrm{~g} / \mathrm{L}$. This effect was hypothesized to be related to the heterogeneous packing of the fibrils, but also here, no complete explanation was found. These two studies highlight both the difficulty in characterization of the process and final material, but also the need for more understanding of the effects of concentration variation. Moreover, note that there is no overlap in the concentrations between the two studies, and one uses suspensions that are in a VAS (or hydrogels) (Lundahl et al. 2016), while the other uses flowing suspensions (Mittal et al. 2019).

Now, let us look at the study coming closest to the dashed line of the blue markers, N (Kim et al. 2019). Kim et al. ( 2019), started with a 20 g/L TEMPO CNF and could produce filaments, N1 and N2, with similar stiffness (23 GPa and $24 \mathrm{GPa}$ ) but varying strength (370 MPa and $490 \mathrm{MPa}$ ) by decreasing the nozzle size. Where the decrease in nozzle size resulted in stronger filaments. The small nozzle was then used in combination with a drawing step to produce N3 with stiffness of $37 \mathrm{GPa}$. The interesting filaments however, are $\mathrm{N} 1$ and $\mathrm{N} 2$ with similar stiffness and varying strength, could this be due to alignment, size of filaments, or maybe homogeneity? The alignment was unfortunately not measured for these cases, however, other studies have shown that the stiffness and alignment are linked, therefore, since the stiffness is the same, the alignment is ruled out. The size of the filament can be the cause but was also not presented in the paper, it is well known that thinner filaments result in stronger filaments, Monteiro et al. (2011). 
Alternatively, could this be the result of enough shear and elongational forces to break up the connected network with the smaller nozzle compared with the larger and thereby form a more homogeneous filament? Further investigations are of course needed, but the results presented by Kim et al. (2019) may indicate that entangled $\mathrm{CNF}$ at rest, resulting in a VAS state, could be forced into an aligned state where a homogeneous entanglement is possible at higher concentrations, proposed by Fall et al. (2013).

Considering the results presented here, it is proposed that the following three conditions must be achieved, and should be characterized, to produce an ideal CNF-filament:

1. The filament must be possible to handle after exiting the spinneret, unless a supporting structure is used.

2. The particle alignment inside the filament must be possible to control.

3. The filament should consist of homogeneously distributed CNF. Any spatial variation will lower the mechanical properties in the final filament.

The first must be achieved for there to be a filament at all and the second condition has been extensively examined and proven (Håkansson 2014, 2015; Håkansson et al. 2016; Mittal et al. 2017, 2018). The third is achieved in studies using the flow focusing nozzle (Håkansson et al. 2014; Mittal et al. 2017, 2018, 2019; Nechyporchuk et al. 2018), but has not been explicitly studied and was therefore targeted in this study. Significant decrease in mechanical properties was observed when a non-homogeneous starting suspension was used. Note that for lab scale film production, only the third point is applicable, and is ensured by starting form a low concentration.

\section{Conclusions}

$\mathrm{CNF}$ at increased concentrations, due to the elongated particle shape of the CNF, forms a VAS, and the suspension is not flowing when acted upon by gravity alone (Nordenström et al. 2017). It is here shown that time independent domains (inhomogeneities) are visible and quantifiable with POM in the VAS for the carboxymethylated $\mathrm{CNF}$ at $12 \mathrm{~g} / \mathrm{L}$. The POM images were analyzed with a Fourier transform to result in a POM Formation spectra and number, quantifying the inhomogeneities. The flowing and non-flowing (VAS) suspensions were used to produce films and filaments, both forms displayed the same trend, decreased mechanical properties when the nonflowing and non-homogeneous suspension was used. It is well known that a well dispersed system results in better performing end materials, surprisingly, the homogeneity of the starting suspension has not previously been investigated for filament spinning from CNF before. Here, strong indications that the homogeneity is directly influencing the mechanical properties are presented. Therefore, it is highly recommended to ensure the homogeneity in future filament and film studies. Naturally, this does not only apply to CNF, but also to other types of nanoparticles, such as carbon nanotubes, graphene and spider silk proteins.

\section{Materials and methods}

\section{CNF preparation}

The material used in these experiments were carboxymethylated CNF to a degree of substitution of 0.1, see Wågberg et al. (1987) for details. The starting pulp was a softwood sulphite dissolving pulp (Domsjö Dissolving plus) from Domsjö Fabriker AB, Sweden. After the carboxymethylation, the pulp was homogenized (Microfluidizer M-110EH, Microfluidics Corp., USA) four times at 1700 bar at $10 \mathrm{~g} / \mathrm{L}$, and thereafter four more times at $5 \mathrm{~g} / \mathrm{L}$, by two serial reaction chambers with smallest dimension $200 \mu \mathrm{m}$ and $100 \mu \mathrm{m}$, respectively.

Three different samples where studied, see Fig. 1a:

1. Original CNF dispersion diluted from 5 to $3 \mathrm{~g} / \mathrm{L}$ by magnetic stirrer (flowing).

2. Original CNF dispersion concentrated from 5 to $12 \mathrm{~g} / \mathrm{L}$ by evaporation with a magnetic stirrer (non-flowing).

3. Sample 2) diluted from 12 to $3 \mathrm{~g} / \mathrm{L}$ by magnetic stirrer (flowing).

\section{Rheological measurement}

The rheological frequency sweep measurements were performed with a Kinexus stress controlled rotational 
rheometer (Malvern Instruments, UK) and the software iSpace (Malvern Instruments, UK). A concentric cylinder in metal with smooth surfaces was used, also known as bob and cup, according to standard ISO3,219/DIN 53,019. The temperature was set to $25{ }^{\circ} \mathrm{C}$, the strain $5 \%$, the minimum integration time $10 \mathrm{~s}$ and frequency range $0.01-1 \mathrm{~Hz}$ for all measurements. The samples were allowed to equilibrate for at least 2 min before the measurement started after being loaded into the cylinder.

\section{Film preparation}

Production of films from $3 \mathrm{~g} / \mathrm{L}$ : First, the suspension was diluted to $1 \mathrm{~g} / \mathrm{L}$ using a magnetic stirrer overnight. The obtained suspension was thereafter degassed for one hour. Films were prepared through vacuum filtration, using $0.65 \mu \mathrm{m}$ DVPP filters (supplied by Millipore), followed by drying under restrained conditions in an oven for $7 \mathrm{~h}$ at $50{ }^{\circ} \mathrm{C}$.

Production of films from $12 \mathrm{~g} / \mathrm{L}$ : To create a film without bubbles the non-flowing suspension was centrifuged on a $0.65 \mu \mathrm{m}$ DVPP filter at $2000 \mathrm{~g}$ for 15 min with a Heraus, Multifuge 3L. An uneven layer was formed on top of the filter, but to achieve a uniform cake of around $2 \mathrm{~mm}$ in thickness, a Teflon coated knife was used to remove excess material. The cake was then dewatered with vacuum in the same way as above.

\section{Spinning}

The suspensions were loaded into a syringe attached to a syringe pump (WPI, Al-4000). The syringe was connected via a hose to a nozzle (TECDIA, ARQ) having a circular exit cross section with $200 \mu \mathrm{m}$ in diameter. The nozzle was submerged in a flowing bath with $\mathrm{pH}$ adjusted to 1 with $\mathrm{HCl}$. The syringe pump was set to $0.5 \mathrm{ml} / \mathrm{min}$. The wet filaments were picked out of the bath and constrained in the two ends during the drying in ambient conditions.

\section{Polarized optical microscopy}

A (Carl Zeiss Axioplan) microscope with crossed polarizers and a camera (ProgRes Jenoptik, Jena) was used to take images $(1360 \times 1024$ pixels 24 bit RGB, TIFF) of the samples. If no samples are placed between the crossed polarizers, no light will pass through the second polarizer and a completely black image is seen. If a sample is placed between the crossed polarizers, the sample could turn the polarisation of the light and features can be detected after the second polarizer. Samples with aligned CNF has been seen to turn the polarisation of light (Håkansson et al. 2014) and more light is detected if the alignment is higher.

The flowing and non-flowing samples were squeezed to a thin layer between a glass slide and a microscopy glass, while the films and filaments were observed as was.

\section{Formation}

Images have been evaluated with the software STFIMottling Expert v1.34. The software divides each image into squares, here, 6 squares of $0.64 \times 0.64$ $\mathrm{mm}^{2}$ (half of the smallest length of the image). Each square is then Fourier transformed in two dimensions. Thereafter, the difference to the mean of the transformed signal in each predefined interval (multiples of $0.64 \mathrm{~mm}$ in this case) is calculated and output. The error-bars correspond to the standard deviation from 10 images for the non-flowing sample and films while 5 images were used for the flowing suspensions.

Tensile testing of films

The tensile properties of the films were investigated with an MTS tensile tester in combination with a Teststar ISS controller (MTS Systems Corp., USA). The films were conditioned for at least $24 \mathrm{~h}$ prior to the tests in the same environment as the test were performed, $50 \% \mathrm{RH}$ and $23{ }^{\circ} \mathrm{C}$. A rectangular shape of the test specimen were used with length of $45 \mathrm{~mm}$ and width of $5 \mathrm{~mm}$. The gap distance was $30 \mathrm{~mm}$ and the speed was set to $0.5 \mathrm{~mm} / \mathrm{s}$. At least 6 specimens per sample were measured.

Tensile testing of filaments

A tensile tester (Dia-Stron Ltd., Andover, UK) consisting of an automated sample loader (ALS1500) and a linear extensometer (LEX820) was used to perform tensile tests on the filaments. The filaments were kept at $50 \% \mathrm{RH} / 23{ }^{\circ} \mathrm{C}$, for at least $24 \mathrm{~h}$ before conducting the measurements. The cross section of each sample was measured with a combination of Scanning 
Electron Microscopy images and optical microscopy. The distance between the grips holding the strips was $12 \mathrm{~mm}$ and the grips moved at a speed of $0.1 \mathrm{~mm} / \mathrm{s}$ during the measurements. At least 10 filaments were tested for each sample.

Wide angle x-ray scattering

Wide Angle X-ray Scattering (WAXS) measurements were performed in a similar manner as already described in Ottesen et al. (2019), but the description will also be included here for calarity. An Anton Paar SAXSpoint 2.0 system (Anton Paar, Graz, Austria) equipped with a Microsource X-ray source $(\mathrm{Cu} \mathrm{K \alpha}$ radiation, wavelength $0.15418 \mathrm{~nm}$ ) and a Dectris $2 \mathrm{D}$ CMOS Eiger R $1 \mathrm{M}$ detector with $75 \mu \mathrm{m}$ by $75 \mu \mathrm{m}$ pixel size was used. All measurements were performed with a beam size of approximately $500 \mu \mathrm{m}$ diameter, at ambient temperature with a beam path pressure at about 1-2 mBar. The sample to detector distance (SDD) was $111 \mathrm{~mm}$ during the measurements. A bundle of $\sim 10$ filaments for each sample were mounted on the Sampler for Solids $10 \times 10 \mathrm{~mm}$ (Anton Paar, Graz, Austria) holder. The Sampler was then mounted on a VarioStage (Anton Paar, Graz, Austria). The samples were exposed to vacuum during measurement. For each sample 24 frames each of 30 minutes duration were read from the detector, giving a total measurement time of 12 hours per sample. For all samples the transmittance was determined and used for scaling of intensities. The software used for instrument control was SAXSdrive version 2.01.224 (Anton Paar, Graz, Austria), and post-acquisition data processing was performed using the software SAXSanalysis version 3.00.042 (Anton Paar, Graz, Austria).

Orientation indexes $\left(f_{c}\right)$ of the samples were determined according to equation:

$f_{c}=\left(180^{\circ}-\mathrm{FWHM}\right) / 180^{\circ}$,

where FWHM is full width at half maximum of the azimuthal profile of the (200) signal (Iwamoto et al. 2011). The order parameter of the samples was determined by azimuthal integration of the angle $\varphi$ of the (200) signal as in Håkansson et al. (2014):

$S=\left\langle\frac{3}{2} \cos ^{2} \varphi-\frac{1}{2}\right\rangle$.
Acknowledgments Åsa Engström, AnneMarie Runebjörk, Shadi Samadnouri, Anita Teleman and Anders Reiman are thanked for excellent supporting work. Fredrik Lundell, Andreas Fall, Tiffany Abitbol, Daniel Söderberg, Tobias Köhnke, Oleksandr Nechyporchuk and Bengt Hagström are greatly acknowledged for fruitful discussions. BillerudKorsnäs, Borregaard, Fibria, Hansol Paper, Holmen, ITC, Mercer, Storaenso, Södra, Swedish Foundation for Strategic Research and FORMAS Project No. 2014-183 are acknowledged for their financial contribution.

Author contributions KMOH came up with the original idea, performed the experiments together with laboratory staff, analyzed the experimental data and wrote the manuscript.

Funding Open access funding provided by RISE Research Institutes of Sweden.

\section{Compliance with ethical standards}

Conflict of interest The author declare that they have no conflict of interest.

Open Access This article is licensed under a Creative Commons Attribution 4.0 International License, which permits use, sharing, adaptation, distribution and reproduction in any medium or format, as long as you give appropriate credit to the original author(s) and the source, provide a link to the Creative Commons licence, and indicate if changes were made. The images or other third party material in this article are included in the article's Creative Commons licence, unless indicated otherwise in a credit line to the material. If material is not included in the article's Creative Commons licence and your intended use is not permitted by statutory regulation or exceeds the permitted use, you will need to obtain permission directly from the copyright holder. To view a copy of this licence, visit http://creativecommons.org/licenses/by/4.0/.

\section{References}

Aulin C, Gällstedt M, Lindström T (2010) Oxygen and oil barrier properties of microfibrillated cellulose films and\&nbsp;coatings. Cellulose 17:559-574. https://doi.org/ 10.1007/s10570-009-9393-y

Behabtu N et al (2013) Strong, light, multifunctional fibers of carbon nanotubes with ultrahigh\&nbsp;conductivity. Science 339:182-186. https://doi.org/10.1126/science. 1228061

Brouzet C, Mittal N, Söderberg LD, Lundell F (2018) Sizedependent orientational dynamics of Brownian nanorods. ACS Macro Lett 7:1022-1027. https://doi.org/10.1021/ acsmacrolett.8b00487

Brouzet C, Mittal N, Lundell F, Söderberg LD (2019) Characterizing the orientational and network dynamics of polydisperse nanofibers on\&nbsp;the nanoscale. Macromolecules 52:2286-2295. https://doi.org/10.1021/ acs.macromol.8b02714 
Denn MM (1983) Fiber Spinn. In: Pearson JRA, Richardsson SM (eds) Computational analysis of polymer processing. Springer, Dordrecht, pp 179-216. https://doi.org/10.1007/ 978-94-009-6634-5_6

Dinwoodie JM (2000) Timber: its nature and behaviour. Taylor \& Francis, London

Eichhorn SJ et al (2009) Review: current international research into cellulose nanofibres and nanocomposites. J Mater Sci 45:1-33. doi:https://doi.org/10.1007/s10853-009-3874-0

Fall AB, Lindström SB, Sundman O, Ödberg L, Wågberg L (2011) Colloidal stability of aqueous nanofibrillated\&nbsp;cellulose dispersions . Langmuir 27:11332-11338. https://doi.org/10.1021/la201947x

Fall AB, Lindström SB, Sprakel J, Wågberg L (2013) A physical cross-linking process of cellulose nanofibril gels with shear-controlled fibril orientation. Soft Matter 9:1852-1863. doi:https://doi.org/10.1039/c2sm27223g

Foster EJ et al (2018) Current characterization methods for cellulose nanomaterials. Chem Soc Rev 47:2609-2679. doi:https://doi.org/10.1039/c6cs00895j

Fujisawa S, Okita Y, Fukuzumi H, Saito T, Isogai A (2011) Preparation and characterization of TEMPO-oxidized cellulose nanofibril films with free carboxyl groups. Carbohyd Polym 84:579-583. doi:https://doi.org/10.1016/j.carbpol. 2010.12.029

Fukuzumi H, Saito T, Iwata T, Kumamoto Y, Isogai A (2009) Transparent and high gas barrier films of cellulose nanofibers prepared by\&nbsp;TEMPO-mediated oxidation. Biomacromolecules 10:162-165. https://doi.org/10.1021/ bm801065u

Geng L et al (2018) Understanding the mechanistic behavior of highly charged cellulose nanofibers in aqueous\&nbsp;systems. Macromolecules 51:1498-1506. https://doi.org/10.1021/acs.macromol.7b02642

Geng L, Chen B, Peng X, Kuang T (2017) Strength and modulus improvement of wet-spun cellulose I filaments by sequential physical and chemical cross-linking. Mater Des 136:45-53. https://doi.org/10.1016/j.matdes.2017.09.054

Ghasemi S, Tajvidi M, Bousfield D, Gardner D, Gramlich W (2017) Dry-spun neat cellulose nanofibril filaments: influence of drying temperature and nanofibril structure on\&nbsp;filament properties. Polymers 9:392. https://doi. org/10.3390/polym 9090392

Gonzalez RC, Woods RE (1993) Digiatal image processing. World student series. Addison-Wesley Publishing Company, Boston

Håkansson KMO et al (2014) Hydrodynamic alignment and assembly of nanofibrils resulting in strong cellulose filaments. Nat Commun 5:4018. doi:https://doi.org/10.1038/ ncomms 5018

Håkansson KMO (2015) Online determination of anisotropy during cellulose nanofibril assembly in a flow focusing device. RSC Adv 5:18601-18608. doi:https://doi.org/10. 1039/c4ra12285b

Håkansson KMO, Lundell F, Prahl-Wittberg L, Soderberg LD (2016) Nanofibril alignment in flow focusing: measurements and calculations. J Phys Chem B 120:6674-6686. https://doi.org/10.1021/acs.jpcb.6b02972

Henriksson M, Berglund LA, Isaksson P, Lindström T, Nishino $\mathrm{T}$ (2008) Cellulose nanopaper structures of high toughness.
Biomacromolecules 9:1579-1585. https://doi.org/10.1021/ bm800038n

Hooshmand S, Aitomaki Y, Norberg N, Mathew AP, Oksman K (2015) Dry-spun single-filament fibers comprising solely cellulose nanofibers from bioresidue. ACS Appl Mater Inter 7:13022-13028. https://doi.org/10.1021/acsami. 5 b03091

Hooshmand S, Aitomäki Y, Berglund L, Mathew AP, Oksman K (2017) Enhanced alignment and mechanical properties through the use of hydroxyethyl cellulose in solvent-free native cellulose spun filaments. Compos Sci Technol 150:79-86. doi:https://doi.org/10.1016/j.compscitech. 2017.07.011

Iwamoto S, Isogai A, Iwata T (2011) Structure and mechanical properties of wet-spun fibers made from natural\&nbsp;cellulose nanofibers. Biomacromolecules 12:831-836. https://doi.org/10.1021/bm101510r

Johansson P-A, Norman B (1996) Methods for evaluating formation, print unevenness and gloss variations developed at STFI. In: Process and product quality conference, Cincinnati, OH, USA. Tappi Press, pp 139-145

Kafy A, Kim HC, Zhai L, Kim JW, Hai LV, Kang TJ, Kim J (2017) Cellulose long fibers fabricated from cellulose nanofibers and its strong and tough characteristics. Sci Rep 7:17683. doi:https://doi.org/10.1038/s41598-017-17713-3

Kerekes RJ, Schnell CJ (1992) Characterization of fibre flocculation regimes by a crowding factor. J Pulp Pap Sci 18:32-38

Kim HC, Kim D, Lee JY, Zhai L, Kim J (2019) Effect of wet spinning and stretching to enhance mechanical properties of cellulose nanofiber filament. Int J Precis Eng Manuf GT 6:567-575. https://doi.org/10.1007/s40684-019-00070-z

Kinahan ME et al (2011) Tunable silk: using microfluidics to fabricate silk fibers with\&nbsp;controllable properties. Biomacromolecules 12:1504-1511. https://doi.org/10. $1021 / \mathrm{bm} 1014624$

Klemm D et al (2018) Nanocellulose as a natural source for groundbreaking applications in materials science: today's state . Mater Today 21:720-748. https://doi.org/10.1016/j. mattod.2018.02.001

Klemm D, Kramer F, Moritz S, Lindstrom T, Ankerfors M, Gray D, Dorris A (2011) Nanocelluloses: a new family of naturebased materials. Angew Chem Int Edit 50:5438-5466. doi:https://doi.org/10.1002/anie.201001273

Lundahl MJ, Cunha AG, Rojo E, Papageorgiou AC, Rautkari L, Arboleda JC, Rojas OJ (2016) Strength and water interactions of cellulose I filaments wet-spun from cellulose nanofibril. Hydro Sci Rep 6:30695. https://doi.org/10. 1038/srep30695

Mason SG (1950) The flocculation of pulp suspensions and the formation of\&nbsp;paper. Tappi J 33:440-444

Mertaniemi $\mathrm{H}$ et al (2016) Human stem cell decorated nanocellulose threads for\&nbsp; biomedical applications. Biomaterials 82:208-220. https://doi.org/10.1016/j. biomaterials.2015.12.020

Mittal N et al (2017) Ultrastrong and bioactive nanostructured bio-based composites. ACS Nano 11:5148-5159. https:// doi.org/10.1021/acsnano.7b02305

Mittal N et al (2018) Multiscale control of nanocellulose assembly: transferring remarkable nanoscale fibril 
mechanics to macroscale fibers. ACS Nano 12:6378-6388. https://doi.org/10.1021/acsnano.8b01084

Mittal N, Benselfelt T, Ansari F, Gordeyeva K, Roth SV, Wagberg L, Soderberg LD (2019) Ion-specific assembly of strong, tough, and stiff biofibers . Angew Chem Int Edit 58:18562-18569. https://doi.org/10.1002/anie.201910603

Mohammadi P, Toivonen MS, Ikkala O, Wagermaier W, Linder MB (2017) Aligning cellulose nanofibril dispersions for tougher fibers. Sci Rep 7:11860. doi:https://doi.org/10. 1038/s41598-017-12107-x

Monteiro SN, Lopes FPD, Barbosa AP, Bevitori AB, Silva ILAD, Costa LLD (2011) Natural lignocellulosic fibers as engineering materials: an overview . Metall Mater Trans A 42:2963-2974. https://doi.org/10.1007/s11661-011-07896

Naderi A, Lindström T, Pettersson T (2014) The state of carboxymethylated nanofibrils after homogenization-aided dilution from concentrated suspensions: a\&nbsp;rheological perspective. Cellulose 21:2357-2368. https://doi.org/ 10.1007/s10570-014-0329-9

Naderi A, Lindström T, Sundström J (2014) Carboxymethylated nanofibrillated cellulose:\&nbsp;rheological studies. Cellulose 21:1561-1571. https://doi.org/10.1007/s10570-0140192-8

Nechyporchuk O, Håkansson KMO, Lundell GowdaVK, Hagström F, Köhnke B (2018) Continuous assembly of cellulose nanofibrils and nanocrystals into strong macrofibers through microfluidic spinning. Adv Mater Technol. https:// doi.org/10.1002/admt.201800557

Nordenström M, Fall A, Nyström G, Wågberg L (2017) Formation of colloidal nanocellulose glasses gels. Langmuir 33:9772-9780. https://doi.org/10.1021/acs.langmuir. $7 \mathrm{~b} 01832$

Ottesen V, Larsson PT, Chinga-Carrasco G, Syverud K, Gregersen ØW (2019) Mechanical properties of cellulose nanofibril films: effects of crystallinity and its modification by treatment with liquid\&nbsp; anhydrous ammonia. Cellulose 26:6615-6627. https://doi.org/10.1007/s10570-01902546-2

Pääkkö M et al (2007) Enzymatic hydrolysis combined with mechanical shearing and high-pressure homogenization for nanoscale cellulose fibrils and strong gels. Biomacromolecules 8:1934-1941

Rosen T, Mittal N, Roth SV, Zhang P, Lundell F, Soderberg LD (2020) Flow fields control nanostructural organization in semiflexible networks. Soft Matter. https://doi.org/10. 1039/c9sm01975h

Saito T, Nishiyama Y, Putaux J-L, Vignon M, Isogai A (2006) Homogeneous suspensions of individualized microfibrils from TEMPO-catalyzed oxidation of\&nbsp;native cellulose. Biomacromolecules 7:1687-1691. https://doi.org/10. 1021/bm060154s

Saxena IM, Brown RM Jr (2005) Cellulose biosynthesis: current views and evolving concepts. Ann Bot 96:9-21. doi:https:// doi.org/10.1093/aob/mci155

Shen $\mathrm{Y}$ et al (2016) High velocity dry spinning of nanofibrillated cellulose (CNF) filaments on an adhesion controlled surface with\&nbsp;low friction. Cellulose 23:3393-3398. https://doi.org/10.1007/s10570-016-1044-5

Syverud K, Stenius P (2008) Strength and barrier properties of\&nbsp;MFC films. Cellulose 16:75-85. https://doi.org/ 10.1007/s10570-008-9244-2

Toivonen MS, Kurki-Suonio S, Wagermaier W, Hynninen V, Hietala S, Ikkala O (2017) Interfacial polyelectrolyte complex spinning of cellulose nanofibrils for advanced\&nbsp; bicomponent fibers. Biomacromolecules 18:1293-1301. https://doi.org/10.1021/acs.biomac. $7 \mathrm{~b} 00059$

Torres-Rendon JG, Schacher FH, Ifuku S, Walther A (2014) Mechanical performance of macrofibers of cellulose and chitin nanofibrils aligned by wet-stretching: a\&nbsp;critical comparison. Biomacromolecules 15:2709-2717. https://doi.org/10.1021/bm500566m

Usov I et al (2015) Understanding nanocellulose chirality and structure-properties relationship at the single fibril level. Nat Commun 6:7564. doi:https://doi.org/10.1038/ ncomms 8564

Wågberg L, Winter L, Ödberg L, Lindström T (1987) On the charge stoichiometry upon adsorption of a cationic polyelectrolyte on cellulosic materials. Colloids Surf 27:163-173

Walther A, Timonen JV, Diez I, Laukkanen A, Ikkala O (2011) Multifunctional high-performance biofibers based on wetextrusion of renewable native cellulose nanofibrils. Adv Mater 23:2924-2928. doi:https://doi.org/10.1002/adma. 201100580

Yao J, Chen S, Chen Y, Wang B, Pei Q, Wang H (2017) Macrofibers with high mechanical performance based on aligned bacterial cellulose nanofibers. ACS Appl Mater Inter 9:20330-20339. https://doi.org/10.1021/acsami. $6 \mathrm{~b} 14650$

Publisher's Note Springer Nature remains neutral with regard to jurisdictional claims in published maps and institutional affiliations. 